\title{
Audio and Audiovisual Cueing in Visual Search: Effects of Target Uncertainty and Auditory Cue Precision
}

\author{
Hugo Bertolotti and Thomas Z. Strybel \\ California State University, Long Beach, \\ Center for the Study of Advanced Aeronautics Technologies \\ 1250 N Bellflower Blvd. Long Beach, CA 90840, USA \\ hbertolo11@yahoo.com, tstrybel@csulb.edu
}

\begin{abstract}
Auditory spatial cue accuracy and target uncertainty were examined within visual search. Participants identified a visual target to be present or absent under various target percentage conditions $(25 \%, 50 \%$, \& $100 \%)$ with either an auditory cue which was spatially coincident with or displaced $4^{\circ}$ or $8^{\circ}$ (vertical or horizontal) from the target, or both an auditory and visual cue (circle $6.5^{\circ}$ radius; identifying the local-target-area surrounding the target). Within the auditory cue condition, horizontal displacement was a greater detriment to target present search times than vertical displacement, regardless of error magnitude or target percentage. When provided an audiovisual cue, search times decreased $25 \%$ for present targets, and as much as $300 \%$ for absent targets. Furthermore, within audiovisual cue condition, while present target search times decreased with target percentage, absent target search times increased with target percentage. Cue condition and target uncertainty driven search strategies are discussed, with recommended design requirements and research implementations.
\end{abstract}

Keywords: Visual Search, Audio Cue, Audiovisual Cue, Auditory Cue Precision, Target Uncertainty, False Alarm.

\section{Introduction}

In recent decades, technological advancements have made virtual environments and complex audiovisual displays a possible design solution for aircraft cockpits, automobiles, and other complex work environments. Environments such as these require operators to take in and analyze a consistent influx of information and data, and make critical decisions based upon the information. Moreover, with the majority of information presented to the operator being visual, inundation of the already overloaded visual channel is possible. These complex work environments might avoid visual overload by providing some information via other sensory channels, for example, auditory. The information provided from the auditory channel can be either redundant with information presented visually (creating an audiovisual display), or specific to the auditory channel alone (creating an auditory display). 
Research concerning auditory spatial cueing in visual search has demonstrated several benefits of providing operators with spatially coincident auditory cues -auditory cues provided at a specific elevation and azimuth in space - in both laboratory and applied settings. Within a controlled laboratory environment, coincident auditory spatial cues have been shown to significantly reduce search times required to locate and identify a specified target during a visual search task [1] [2]. In applied settings, simulated 3D auditory spatial cues (simulated interaural difference cues and spectral shape cues provided via headphones) improved target acquisition, traffic detection and avoidance, and visual workload [3] [4] [5]. It is important to note that while providing an auditory spatial cue may be advantageous, the magnitude of the benefits provided is contingent upon the characteristics of the auditory cue and the visual task itself.

One important auditory cue characteristic, auditory cue precision (measured as the distance between the location of the audio cue and the location of the visual target), has been demonstrated to significantly affect target identification [6] [7] [8]. Research has shown that increasing error magnitude displacement $\left(0^{\circ}\right.$ to $\left.8^{\circ}\right)$ between a specified target and an auditory spatial cue will significantly increase target search times [7] [8]; however, while search times consistently increased with error magnitude displacement, manipulating error displacement direction produced inconclusive results. Within $\mathrm{Vu}$ et al. [6], vertical displacement of an auditory spatial cue was a greater detriment to target identification than horizontal displacement, whereas Bertolotti and Strybel [7] found horizontal displacement to be more detrimental. Moreover, as the error displacement of auditory spatial cue relative to the target increased, the directional discrepancies between Bertolotti and Strybel, and Vu et al. become larger.

A possibility for the inconsistent dimensional findings may be due to target uncertainty. While a target was present on every trial within $\mathrm{Vu}$ et al. [6], Bertolotti and Strybel [7] not only included trials which contained no targets (false targets), requiring participants to respond "no target" when none was found, but also manipulated the percentage of trials containing no targets within a given block of trials. When false targets were introduced, horizontal displacement became significantly more detrimental to target identification than vertical displacement, regardless of target percentage. In addition, the time required to identify the absence of a target was three times that of a present target, regardless of cue precision. Perhaps, the introduction of false alarms produced alterations in observer search strategies, and it is these search strategy adjustments which lead to discrepancies within the obtained dimensional findings. A second possibility for the dimensional inconsistencies may be due to the differences in visual saliencies of both the local area surrounding the target (local-target-area), and the entire visual search field (global area). While local and global visual saliencies remained equal within Bertolotti and Strybel [7], Vu et al. [6] manipulated local and global visual saliencies via distractor density. Results show when the local-target-area surrounding the target was visually cued via global-local distractor densities, vertically displaced auditory cues produced significantly higher search times than horizontally displaced auditory cues. It appears that as the local target area becomes increasingly visually salient as a 
result of global-local distractor density combinations, facilitation of the localization stage of the visual search process may occur, accounting for the dimensional inconsistencies.

To examine these two possibilities, and to gain a clearer understanding of the inconsistent dimensional findings in the previous literature, the current study observed the effects of auditory spatial cue precision, target uncertainty, and local target area visual saliency on the visual search process.

\section{Method}

\subsection{Participants}

Twelve students ( 5 males, 7 females) with a mean age of 23.92 years $(\mathrm{SD}=3.37$ years) participated in the study. All participants were students from California State University, Long Beach, and reported normal hearing and normal to corrected-tonormal vision. Participants were paid $\$ 60$ for completing the experiment.

\subsection{Apparatus}

The apparatus and materials utilized were identical to that of Bertolotti and Strybel [7]. Positioned at the center of a semi-anechoic room, covered by Markerfoam 10.16 $\mathrm{cm}$ acoustic foam sheets (absorption coefficients exceed .90 for frequencies greater than $250 \mathrm{~Hz}$ ), was a large acoustically transparent projection screen. Figure 1 illustrates a sample of the visual search field consisting of a target $\left(1^{\circ} \mathrm{x} 1^{\circ}\right.$ arrowhead pointing right or left), distractors $\left(1^{\circ} \times 1^{\circ}\right.$ arrowheads pointing up or down), and a visual cue (described later). Contrast between the white targets and distractors and the black screen was held constant at roughly 75\%, with local and global distractordensities remaining constant at $32 \%$. Two data projectors connected to a microprocessor located in an adjacent room were used for presenting the visual stimulus during the experiment.

For the auditory stimulus, Tucker-Davis Technologies' audio modules were used for generating and presenting the $65-\mathrm{dB}$ A-weighted auditory stimulus. The auditory stimulus was a series of $300 \mathrm{~ms}$ broadband noise bursts separated by $100 \mathrm{~ms}$ quiet intervals that remained on until a response was made. Forty-five $7.6 \mathrm{~cm}$ Blaupunkt speakers mounted behind the screen in six circular centric rings produced the auditory stimuli. The positioning of the speakers, measured from the fixation point in the middle of the visual search field, created three distance ranges $\left(12^{\circ}\right.$ to $18^{\circ} ; 24^{\circ}$ to $30^{\circ}$; $36^{\circ}$ to $42^{\circ}$ ) with midpoints of $15^{\circ}, 27^{\circ}$, and $39^{\circ}$. The fixation point consisted of a $1^{\circ} \mathrm{x}$ $1^{\circ}$ crosshair, located at the center of the visual search field.

In order to record participant responses, a four button response box was utilized. The two top buttons were used to identify the target (left button-arrowhead pointing to the left; right button-arrowhead pointing to the right), while either of the two bottom buttons were used to report no target present. 
$92^{\circ}$

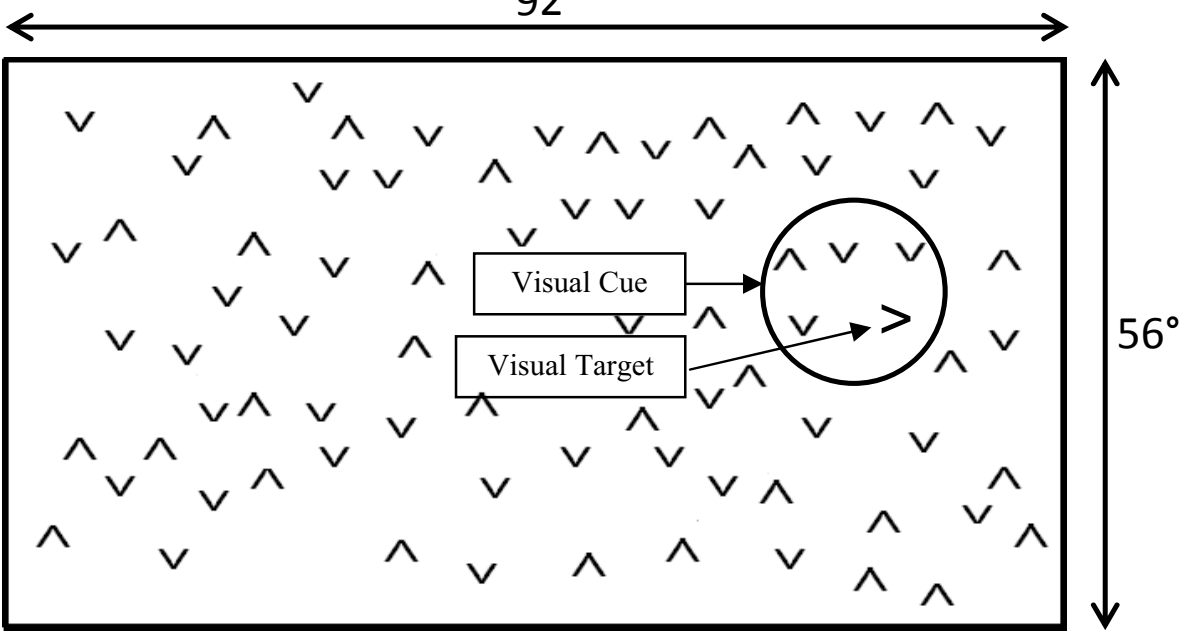

Fig. 1. Visual search field indicating visual cue (circle: $6.5^{\circ}$ radius), and consisting of a visual target (arrowhead pointing right) and distractors (arrowheads pointing up or down)

\subsection{Design}

Four independent variables were manipulated: cue condition, auditory cue error, target percentage, and target distance. Cue condition was the first variable under investigation, and consisted of two levels: auditory cue and audiovisual cue. Within the auditory cue condition, the participant was provided with only an auditory spatial cue which signaled the location of the target. In the audiovisual cue condition, the participant was provided with a visual cue in addition to the auditory spatial cue. Following the local target area dimensions of previous research [8], the visual cue consisted of a circle ( $6.5^{\circ}$ radius) identifying the local target area (Figure 1). Auditory cue error, the second variable under investigation, varied the direction and distance between the auditory spatial cue and the target. There were two displacement directions (horizontal and vertical), and three error magnitude distances $\left(0^{\circ}, 4^{\circ}, \& 8^{\circ}\right)$.

Target percentage was the next independent variable, and consisted of three levels: $25 \%, 50 \%$, and $100 \%$. In the $25 \%$ target percentage condition, $25 \%$ of the trials within a session contained targets. The second target percentage condition provided targets on $50 \%$ of the trials within a given session. The final target percentage condition provided a target on $100 \%$ of the trials. For no target trials, a distractor (arrowhead pointing either up or down) was placed in the exact position where the target would have been.

The final independent variable was target distance from the point of fixation, and there were three distance ranges: $12^{\circ}$ to $18^{\circ}, 24^{\circ}$ to $30^{\circ}$, and $36^{\circ}$ to $42^{\circ}$. These three distance ranges created midpoints of $15^{\circ}, 27^{\circ}$, and $39^{\circ}$ accordingly. Again, a $1^{\circ} \times 1^{\circ}$ crosshair located at the center of the visual search field was considered the point of fixation. It must be mentioned that at the farthest distance from the point of fixation $\left( \pm 39^{\circ}\right)$, horizontal displacement of the auditory cue outside the local target area $\left(8^{\circ}\right)$ 
was possible only in the direction towards the point of fixation due to the close proximity of the speakers to the peripheral edge of the visual search field. The dependent variable for the current study was target and no target search times.

\subsection{Procedure}

Participants were required to complete a screening form and instructed on the purpose of the study before signing the informed consent. Once completed, participants were seated $127 \mathrm{~cm}$ from the projected visual search field and instructed to located and identify whether a visual target was present or absent, and if present, identify the direction it was pointing. A practice session (20 trials) was given in order to familiarize the participant with the experiment and how to respond. After the participant reached a response accuracy rate of $95 \%$, the participant was instructed that the experiment was about to begin, and was provided information regarding the visual cue and target percentage for the current block.

Upon the start of a trial, the participant was required to fixate on the crosshair located at the center of the visual search field containing many X's. After a period of $500 \mathrm{~ms}-1000 \mathrm{~ms}$, the crosshair disappeared, the X's turned into distractors, and a target accompanied by the appropriate cue (auditory or audiovisual) was presented. The participant was instructed to scan the visual search field and report, via the response box, the direction of the target (left or right) or no target. After the response the trial terminated, the visual search field disappeared, and the crosshair positioned at the center of the search field accompanied by the X's reappeared to signify the start of the next trial. If no response was made after 8 seconds, the trial was terminated.

Participants completed a total of six 1-hour blocks, of which three blocks were completed with only an auditory cue and three blocks were completed with an audiovisual cue, in random order. Within each cue condition, the three blocks were further separated by target percentage, resulting in each cue condition containing a separate 1-hour block for each target percentage condition $(25 \%, 50 \%$, \& $100 \%)$. Participants were informed of both cue condition and target percentage prior to starting a block. Within each 1-hour block, three separate 20-minute sessions were completed. Of the three sessions completed within each 1-hour block, the first session was considered practice while the remaining two were analyzed.

\section{Results}

To adjust for skewed distribution of search times, ANOVAs were run using log transformations of search times; however, actual search times are used in figures for ease of interpretation. Any violations of Mauchly's test of sphericity were corrected, and adjusted degrees of freedom from Huynh-Feldt estimates of sphericity were used. Post-hoc analyses were completed with the use of Tukey post-hoc test.

Separate ANOVAs were run for each cue condition due to the large differences in search times between the audiovisual cue condition (target present: $\mathrm{M}=911.99 \mathrm{~ms}$, SEM $=74.8 \mathrm{~ms}$; target absent: $\mathrm{M}=835.375 \mathrm{~ms}, \mathrm{SEM}=69.86 \mathrm{~ms}$ ) and the auditory cue condition (target present: $\mathrm{M}=1134.69 \mathrm{~ms}, \mathrm{SEM}=117.57 \mathrm{~ms}$; target absent: 
$\mathrm{M}=3748.03 \mathrm{~ms}, \mathrm{SEM}=396.32 \mathrm{~ms})$. For the target present trials, two 3 (target percentage: $25 \%, 50 \%$, \& $100 \%$ ) X 3 (target distance: $15^{\circ}, 29^{\circ}, \& 39^{\circ}$ ) X 2 (error direction: horizontal and vertical) X 3 (error magnitude: $0^{\circ}, 4^{\circ}, \& 8^{\circ}$ ) repeated measures analysis of variance (ANOVAs) were run on target present search times for each cue condition (auditory and audiovisual). For the target absent trials, two 2 (target percentage: $25 \%$ \& 50\%) X 3 (target distance: $15^{\circ}, 27^{\circ}, \& 39^{\circ}$ ) X 2 (error direction: horizontal and vertical) X 3 (error magnitude: $0^{\circ}, 4^{\circ}, \& 8^{\circ}$ ) repeated measures analysis of variance (ANOVAs) were run on target absent search times for each cue condition (auditory and audiovisual). ANOVAs run on target absent trials for each cue condition contained only two percentage conditions ( $25 \%$ \& $50 \%$ ), due to a lack of no target trials within the $100 \%$ target percentage condition. Response accuracy was $98.08 \%(S D=1.14 \%)$, and trials which participants time-out were removed prior to analysis.

\subsection{Auditory Cue Condition}

For target present trials, a significant main effect of error direction was obtained $(F(1$, $11)=10.425, p=.012)$. Search times with horizontally displaced cues $(M=1254.79$ $\mathrm{ms}, S E M=80.84 \mathrm{~ms}$ ) were significantly higher than search times with vertically displaced cues $(M=1132.83 \mathrm{~ms}, S E M=63.60 \mathrm{~ms})$. A significant main effect of error magnitude also was obtained for target present trials $(F(2,22)=15.121, p=.001)$, with search times significantly increasing with error magnitude: $0^{\circ}: M=1102.21 \mathrm{~ms}$, $S E M=67.43 \mathrm{~ms} ; 4^{\circ}: M=1165.88 \mathrm{~ms}, S E M=63.55 \mathrm{~ms} ;$ and $8^{\circ}: M=1313.34 \mathrm{~ms}$, $S E M=87.98 \mathrm{~ms}$. No significant main effects were found on target absent latencies within the auditory cue condition.

The significant main effects for target present trials were qualified by a significant error direction $\mathrm{X}$ error magnitude interaction $(F(1.564,17.487)=6.623, p=.022)$, shown in Figure 2a. While search times for target present trials increased with error magnitude for both horizontal and vertical displacement, search times remained consistently higher when the auditory cue was displaced horizontally compared to vertically, particularly at $8^{\circ}$ of error magnitude displacement.

Simple effects analysis revealed a significant simple effect of error direction at both $4^{\circ}(F(1,11)=12.348, p=.005)$ and $8^{\circ}(F(1,11)=10.407, p=.008)$. Horizontal displacement was found to be a significantly greater detriment to target identification than vertical displacement, regardless of error magnitude. To examine the linear relationship between both error directions, as well as the increasing rate of search time, mean search times were regressed against error magnitude. Only horizontal displacement was found to explain a significant portion of the variance in target identification search times (horizontal displacement: $\mathrm{R}^{2}=.97 ; F(1,11)=36.403, p=$ .01 ; vertical displacement: $\mathrm{R}^{2}=.78 ;(F(1,11)=3.581, p=.30)$. Furthermore, a slope of $43.57( \pm 7.22)$ was obtained for horizontal displacement, and $9.213( \pm 4.87)$ for vertical displacement. Thus, for every $1^{\circ}$ increase in horizontal displacement, search times increased by approximately $43 \mathrm{~ms}$, and every $1^{\circ}$ increase in vertical displacement, search times increased by approximately $9 \mathrm{~ms}$. 

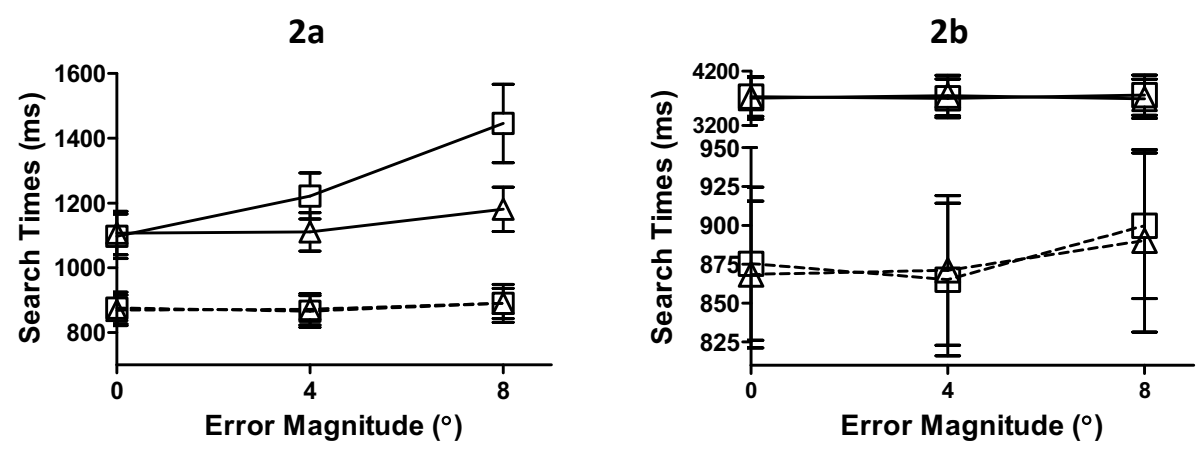

Error Direction - Cue Condition

$\square \quad$ Horizontal - Auditory

$\triangle$ Vertical - Auditory

$-\square--$ Horizontal - Audiovisual
$-\triangle--$ Vertical - Audiovisual

Fig. 2. Search times for auditory and audiovisual cue conditions as a function error direction and error magnitude: a) target present trials b) target absent trials

A significant error magnitude $\mathrm{X}$ target percentage interaction also was obtained for only the target present trials $(F(4,20)=2.98, \mathrm{p}<.05)$. As illustrated in Figure 3, at 25\% target percentage, error magnitude had little effect on search times; however, as target percentage increased, search times increased with error magnitude. Simple effect of error magnitude on target percentage demonstrated a significant simple effect at $50 \%(F(2,22)$ $=21.014, p<.001)$ and $100 \%(F(1.491,16.4)=22.534, p<.001)$. Post-hoc tests found a significant increase in search times at $50 \%$ as error magnitude increased from $0^{\circ}$ to $8^{\circ}$ and $4^{\circ}$ to $8^{\circ}$, and a significant increase in search times at $100 \%$ for every error magnitude increment. Thus, while error magnitude did not affect target present trial search times at $25 \%$, search times significantly increased for every error magnitude increment at both $50 \%$ and $100 \%$, with the exception of the $0^{\circ}$ to $8^{\circ}$ increment at $50 \%$.

$3 a$

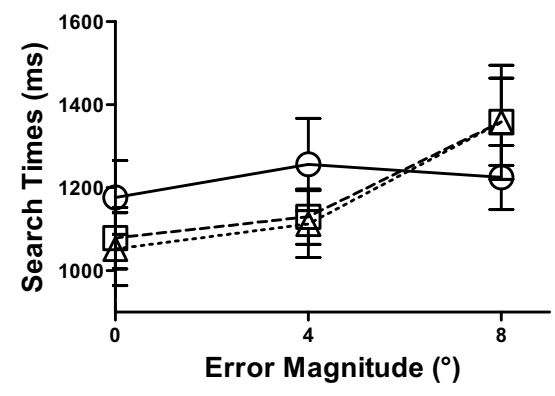

3b

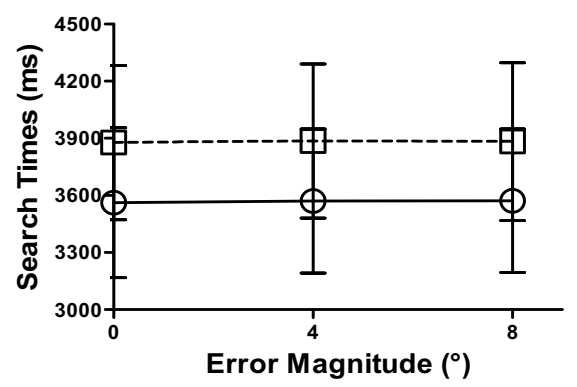

Target Percentage

- $25 \% \quad-$ - 5 - $50 \% \quad-A-100 \%$

Fig. 3. Search times for the auditory cue condition as a function target percentage and error magnitude: a) target present trials b) target absent trials (contains only $25 \%$ and $50 \%$ conditions) 


\subsection{Audiovisual Cue Condition}

For target present trials, a significant main effect of target percentage was obtained $(F(2,11)=10.03, p=.002)$. While search times decreased as target percentage increased (25\%: $\quad M=921.40 \mathrm{~ms} ; S E M=70.32 \mathrm{~ms} ; 50 \%: \quad M=869.09 \mathrm{~ms}, S E M=$ $57.19 \mathrm{~ms}$; 100\%: $M=843.99 \mathrm{~ms}, S E M=56.58 \mathrm{~ms}$ ), post-hoc testing found only one significant difference in search times, $25 \%$ vs. $100 \%$. A significant main effect of target distance was also found for target present trials $(F(2,11)=19.539, p<.001)$. Post-hoc testing determined that as target distance increased, search times significantly increased only at target distances between $15^{\circ}(M=871.26 \mathrm{~ms}, S E M=$ $62.98 \mathrm{~ms})$ vs. $39^{\circ}(M=902.01 \mathrm{~ms}, S E M=58.47 \mathrm{~ms})$, and $27^{\circ}(M=861.21 \mathrm{~ms}, S E M$ $=57.19 \mathrm{~ms}$ ) vs $39^{\circ}$. No significant interactions were obtained for target present trials within the audiovisual cue condition.

For target absent trials, a significant main effect of target percentage was obtained $(F(1,11)=5.951, p=.033)$, with search times significantly increasing with target percentage. A significant main effect of target distance was also found for target absent trials $(F(2,22)=32.624, p<.001)$. Significant increases in search times were found between $15^{\circ}$ vs. 39 , and $27^{\circ}$ vs. $39^{\circ}$. However, between $15^{\circ}$ vs. $27^{\circ}$, search times at $27^{\circ}$ were significantly lower than search times at $15^{\circ}$. A significant main effect of error magnitude was found for target absent trials $(F(2,22)=4.076, p=.031)$. Significant increases in search times were found between $0^{\circ}$ to $4^{\circ}$, and $4^{\circ}$ to $8^{\circ}$. No difference in search times were found as error magnitude increased from $0^{\circ}$ to $8^{\circ}$.
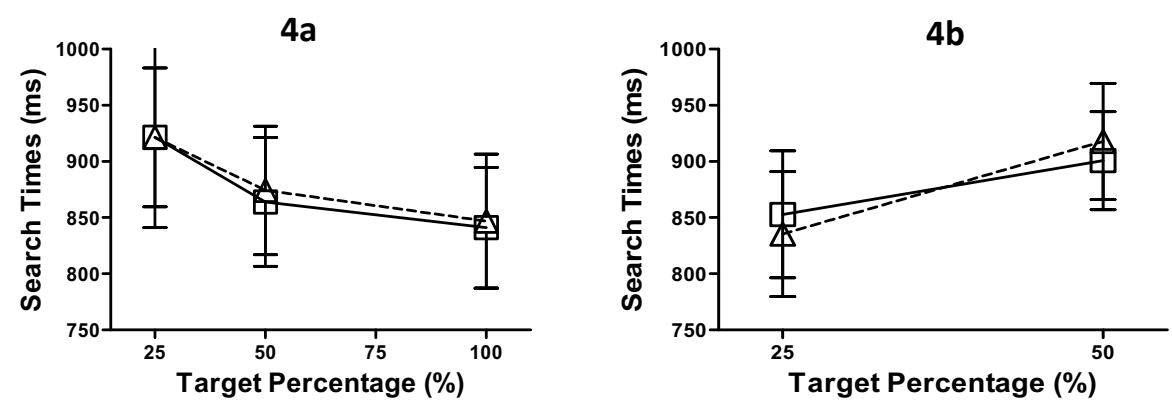

Error Direction

$\square$ Horizontal $\quad-\triangle--\cdot$ Vertical

Fig. 4. Search times for the audiovisual cue condition as a function error direction and target percentage: a) target present trials b) target absent trials

For target absent trials, a significant target percentage $\mathrm{X}$ error direction interaction was obtained (target present: $F(2,22)=.506, p=.614$; target absent: $F(1,11)=$ 5.07, $p=.046$ ). As Figure 4 illustrates, search times increased with target percentage at both horizontal and vertical displacement. Simple effects of target percentage for error direction demonstrated a marginally significant simple effect of target percentage for horizontal displacement $(F(1,11)=3.642, p=.083)$, and a significant main effect of target percentage for vertical displacement $(F(1,11)=7.876, p=.017)$. 
Search times increased with target percentage at both horizontal and vertical displacement, but the rate of increase was higher with vertical displacement. Furthermore, while horizontal displacement was a greater detriment to search times at $25 \%$, vertical displacement was a greater detriment to search times at $50 \%$. Simple effects of error direction for target percentage were examined, and a significant simple effect of error direction was obtained only at $25 \%(F(1,11)=8.699, p=.013)$. Horizontal displacement was a significantly greater detriment to search times than vertical displacement. For the target present trials (Figure 4a), while search times decreased with target percentage at both horizontal and vertical displacement, these were non-significant.

\section{Discussion}

Examining the dimensional findings within the current study, the results appear to be consistent with Bertolotti and Strybel [7]: horizontal displacement of an auditory cue was a greater detriment to the identification of a visual target than vertical displacement. Furthermore, within both the current and previous research [7], horizontally displacing an auditory cue either $4^{\circ}$ or $8^{\circ}$ from a visual target produced higher search times than vertically displacing an auditory cue either $4^{\circ}$ or $8^{\circ}$ from a visual target. Thus, an auditory cue displaced horizontally within the local-target-area increased search times more than a vertically displaced auditory cue outside the localtarget-area. Given that this interaction did not depend on target percentage or target distance, it may reflect differences in localization between the horizontal and vertical plane; specifically, higher auditory spatial acuity in the horizontal plane compared to the vertical plane [9].

As for the first possibility of introducing target uncertainty, the current study found that when target uncertainty was introduced, horizontal displacement of an auditory cue continued to be a greater detriment to visual target identification than vertical displacement, regardless of error magnitude. Moreover, horizontal displacement was a greater detriment to target identification when target percentage was $100 \%$, which suggests that target uncertainty may not be responsible for the incongruent directional displacement findings. In addition, within both the current study and Bertolotti and Strybel [7], search times significantly increased as the auditory cue error and target percentage increased. Therefore, it is suggested that search strategies for a visual target assisted by only an auditory cue are affected by target uncertainty in that as the likelihood of a target increased, greater dependence on the auditory cue for visual target identification was shown, which lead to a greater effect of auditory cue directional displacement. For the directional displacement when no visual target was present, the current study found an effect of directional displacement only as both target percentage and error magnitude increased.

With regards to the second possibility, the findings from the current study are consistent with the previous literature in that increasing local-target-area visual saliency significantly improves the visual search of a target [6] [8]. When provided with a reliable local-target-area visual cue and a variably displaced auditory cue, 
search times decreased by approximately $25 \%$ compared to search times with a variable displaced auditory cue alone. However, while $\mathrm{Vu}$ et al. [6] found variable vertical displacement of the auditory cue outside the local-target-area to be more of a detriment to the visual search process when the local-target-area was visually salient, the current study found the no effect of auditory cue displacement when the localtarget-area was visually cued. Regardless of the error magnitude or directional displacement of the auditory cue, providing a visually salient local-target-area significantly improved visual target identification. It must be noted that while $\mathrm{Vu}$ et al. [6] varied global-local distractor-densities to manipulate the visual saliency of the local-target-area, the saliency of the local-target-area within the current study was varied by either providing a visual cue which accurately and consistently cued the local-target-area, or providing no visual cue. Possibly the high saliency of the localtarget-area visual cue within the current study rendered the directional displacement of the auditory cue ineffective, hindering the ability to reconcile the incongruent findings within Bertolotti and Strybel [7] and Vu et al. [6].

One possible explanation for the ineffectiveness of auditory cue precision with audiovisual cues is that participants used the unreliable auditory cue to identify the hemi-field in which the target was located, while using the visual cue to determine the local-target-area and target location. This is suggested since providing a visually cued local-target-area was found to not only significantly improve target identification [6] [8], but also rendered the effect of auditory cue displacement less effective. Additional evidence for this possibility is that search times for targets assisted by an audiovisual cue were found to increase as target distance increased. Thus, it is suggested that while the auditory cue provided the direction in which the target was located compared to the center of the visual search field, the visual cue provide the local-target-area visual saliency needed to identify the local-target-area, and the target itself.

In summary, these results suggest that the effectiveness of audio spatial cueing systems depend more on precision cueing of horizontal target position compared with vertical target position because of the greater detriment in search latencies found with horizontally displaced cues. Moreover, with production cost much lower for auditory cues required for localization in the horizontal plane (ILDs and ITDs) compared to auditory cues required for localization in the vertical plane (spectral shape cues) [10], minimizing horizontal error would not only improve operator performance, but also would reduce development costs. The current study also has shown that providing an audiovisual cue can significantly improve target search latency compared to only an auditory cue. Furthermore, the benefits of providing an audiovisual cue were greater when participants were required to report no target, with performance improving as much as $300 \%$. Thus, for environments in which false alarms are a possibility, audiovisual cues improve performance over an auditory cue alone.

Acknowledgements. This paper was partially supported by the Center for Human Factors in Advanced Aeronautics Technologies, a NASA University Research Center (NASA cooperative agreement NNX09A66A.) 


\section{References}

1. Perrott, D.R., Cinseros, J., McKinley, R.L., D’Angelo, W.R.: Aurally aided visual search under virtual free-listening conditions. Human Factors 38, 702-715 (1996)

2. Perrott, D.R., Saberi, K., Brown, K., Strybel, T.Z.: Auditory psychomotor coordination and visual search. Perception and Psychophysics 48, 214-226 (1990)

3. Begault, D.R.: Head-up auditory displays for traffic collision avoidance system advisories: A preliminary investigation. Human Factors 35, 707-717 (1993)

4. McKinley, R.L., D’Angelo, W.R., Hass, M.W., Perrott, D.R., Nelson, W.T., Hettinger, L.J., Brickman, B.J.: An initial study of the effects of 3-dimensional auditory cueing on visual target detection. In: Proceedings of the Human Factors and Ergonomics Society, vol. 39, pp. 119-123 (1995)

5. McKinley, R.L., Erickson, M.A.: Flight demonstration of a 3-D auditory display. In: Gilkey, G.H., Anderson, T.R. (eds.) Binaural and Spatial Hearing in Rea; and Virtual Environments, pp. 683-699. Erlbaum, Mahwah, NJ (1997)

6. Vu, K.L., Strybel, T.Z., Proctor, R.D.: Effects of displacement magnitude and direction of auditory cues on auditory spatial facilitation of visual search. Human Factors 49(3), 587599 (2006)

7. Bertolotti, H., Strybel, T.Z.: he effects of auditory cue precision on target detection and identification. Presentation session presented at the meeting Human-Computer Interaction Institution International, Las Veges, NV (2005)

8. Rudmann, D.R., Strybel, T.Z.: Auditory spatial cueing in visual search performance: Effect of local vs. global distractor density. Human Factors 41, 146-160 (1999)

9. Strybel, T.Z., Fujimoto, K.: Minimum audible angles in the horizontal and vertical planes: Effects of stimulus onset asynchrony and burst duration. Journal Acoustical Society of America 108, 3092-3095 (2000)

10. Shinn-Cunningham, B.G.: Spatial auditory displays. In: Karwowski, W. (ed.) International Encyclopedia of Ergonomics and Human Factors, 2nd edn. Taylor and Francis, Ltd., Abington (2002) 